\title{
Exploring the perception of aid organizations' staff about factors affecting management of mass casualty traffic incidents in Iran: a grounded theory study
}

\author{
Javad Bazeli ${ }^{1}$, Aidin Aryankhesal ${ }^{2,3}$, Davoud Khorasani-Zavareh ${ }^{4,5}$
}

${ }^{1} \mathrm{Ph} . \mathrm{D}$. Student in Health in Disasters and Emergencies, School of Health Management and Information Sciences, Iran university of Medical Sciences, Tehran, Iran

${ }^{2}$ Associate Professor, Health Management and Economics Research Center, Iran University of Medical Sciences, Tehran, Iran

${ }^{3}$ Associate Professor, Department of Health Services Management, School of Health Management and Information Sciences, Iran University of Medical Sciences, Tehran, Iran

${ }^{4}$ Associate Professor, Department of Health in Disaster and Emergency, Faculty of Safety, Health and Environment, Shahid Beheshti University of Medical Sciences, Tehran, Iran

${ }^{5}$ Post Doc Research Fellowship, Department of Clinical Sciences and Education, Karolinska Institute, Stockholm, Sweden

\section{Type of article: Original}

\begin{abstract}
Background: Traffic incidents are of main health issues all around the world and cause countless deaths, heavy casualties, and considerable tangible and intangible damage. In this regard, mass casualty traffic incidents are worthy of special attention as, in addition to all losses and damage, they create challenges in the way of providing health services to the victims.

Aim: The present study is an attempt to explore the challenges and facilitators in management of mass casualty traffic incidents in Iran.

Methods: This qualitative grounded theory study was carried out with participation of 14 purposively selected experienced managers, paramedics and staff of aid organizations in different provinces of Iran in 2016. Semistructured interviews were conducted in order to develop the theory. The transcribed interviews were analyzed through open, axial and selective coding.

Results: Despite the recent and relatively good improvements in facilities and management procedure of mass casualty traffic incidents in Iran, several problems such as lack of coordination, lack of centralized and integrated command system, large number of organizations participating in operations, duplicate attempts and parallel operations carried out by different organizations, intervention of lay people, and cultural factors halt provision of effective health services to the victims.

Conclusion: It is necessary to improve the theoretical and practical knowledge of the relief personnel and paramedics, provide public with education about first aid and improve driving culture, prohibit laypeople from intervening in aid operations, and increase quality and quantity of aid facilities.
\end{abstract}

Keywords: Mass casualty incidents, road traffic accidents, car crash, special traffic incidents

\section{Introduction}

Car incidents are among the main issues for health systems all around the world, so much that the death toll of such incidents exceeds one million a year and an additional 20 to 50 million injured (1-3). Moreover, the society has to cover heavy social and economic damage caused by traffic incidents so that around five percent of GNP across low and middle income countries (LMICs) and, on average, three percent of GNP in all countries is devoured by traffic incidents and the resulted casualties $(4,5)$. By 2030, traffic incidents will be the fifth cause of mortality in the world

\section{Corresponding author:}

Aidin Aryankhesal, Iran University of Medical Sciences, Tehran, Iran.

Tel: +98.2188773073, Fax: +98.2188883334, Email: aryankhesal.a@iums.ac.ir

Received: February 23, 2017, Accepted: May 16, 2017, Published: July 2017

iThenticate screening: April 20, 2017, English editing: June 02, 2017, Quality control: June 12, 2017

(C) 2017 The Authors. This is an open access article under the terms of the Creative Commons Attribution-NonCommercialNoDerivs License, which permits use and distribution in any medium, provided the original work is properly cited, the use is non-commercial and no modifications or adaptations are made. 
across all age groups $(6,7)$ and more than $90 \%$ of mortality caused by traffic incidents happen in LMICs (8-10). In Iran, traffic incidents are the first cause of years of life lost (YLL) and the second cause of mortality (11). A mass casualty incident, by definition, is one for which the available facilities and normal operational processes are not enough to manage the incident and the injured people $(12,13)$. The Disaster and Emergency Medical Management Center has introduced a definition for special traffic incidents that includes incidents with more than three deaths or five injured at paramedic stage $(14,15)$. Every day, 11.5 special incidents happen in Iran on average, and more than $90 \%$ of them are traffic incidents (16). Undoubtedly the best way to decrease mortality rate of mass casualty traffic incidents is to prevent them, but complete prevention in LMICs is almost impossible. Still, providing proper paramedic services can be effective in decreasing the losses caused by the incidents (17). Several organizations including Emergency Medical Services (EMS: a subset of the Medical Deputy of the Ministry of Health and Medical Education that is responsible for providing pre-hospital emergency services across the country), the Red Crescent, Police, and Fire Department respond to traffic incidents in Iran. Knowing that about two-thirds of the injured die before arriving at hospitals while one third of them can be saved (18-20), pace and quality of the response to such incidents can be highly effective in reducing the death toll or the resulted disabilities. Because there is a paucity of studies on the effective factors on incident management and ways to improve performance in such conditions, the present paper is an attempt to determine the factors effective on management of mass casualty traffic incidents in Iran based on viewpoints of managers and personnel of aid organizations. Having a clearer picture of these factors and asking the experts for recommendations can enable us to improve the quality of incident management and decrease the death toll or preventable disabilities.

\section{Material and Methods}

\subsection{Study design and participants}

Data gathering was done according to Corbin and Strauss version of grounded theory method. This method is widely recommended for discovering relatively new fields or discovering new viewpoints about the already known fields (21). Fourteen personnel and managers of emergency medical services, the Red Crescent, Police, and Fire departments in Tehran, Khorasan, Semnan, Fars, and Khuzestan Provinces took part in the study between September 2015 and October 2016. The participants were managers and operation personnel with good theoretical and practical experience (three years at least) in aid organizations (Table 1).

Table 1. Specification of the participants

\begin{tabular}{|l|l|l|l|l|}
\hline Organization & Participant No. & Age (year) & Experience & Education level \\
\hline Emergency Medical Service(EMS) & P 1 & 51 & 20 & MD \\
\cline { 2 - 5 } & P 2 & 47 & 10 & MD-PhD \\
\cline { 2 - 5 } & P 3 & 50 & 14 & MD \\
\cline { 2 - 5 } & P 4 & 49 & 8 & MD-PhD \\
\cline { 2 - 5 } & P 5 & 50 & 21 & MD \\
\hline \multirow{5}{*}{ The Red Crescent } & P 6 & 31 & 8 & Bachelor \\
\cline { 2 - 5 } & P 7 & 33 & 10 & Bachelor \\
\cline { 2 - 5 } & P 8 & 36 & 13 & Master \\
\hline Police & P 9 & 55 & 30 & Bachelor \\
\hline & P 10 & 49 & 25 & Bachelor \\
\cline { 2 - 5 } & P 1 & 35 & 10 & Bachelor \\
\hline Fire fighting & P 12 & 37 & 8 & Bachelor \\
\cline { 2 - 5 } & P 13 & 45 & 13 & Bachelor \\
\cline { 2 - 5 } & P 14 & 32 & 11 & Bachelor \\
\hline
\end{tabular}

\subsection{Data collection}

Semi-structured interviews are the most commonly used methods for data gathering in qualitative research in the field of health, which enables the researcher to gather rich information (22). Interviews were conducted in Farsi language and at the workplaces of the participants. Each interview took between 30 to 60 minutes. The participants were briefed about the purpose of the study and then questioned about their experience in management of mass casualty traffic incidents. Research questions included: (I) participants' views about the status quo of managing traffic mass casualty incidents in Iran; and (II) factors effective on management of such incidents. The interviews were recorded and transcribed verbatim after they were listened to several times by the leading author (JB). 


\subsection{Data analysis}

To create a deep insight about the data, the interviews were read several times and then coded using keywords in the text or what was perceived by the researchers. The coding process included open, axial and selective coding (Strauss \& Corbin), through which the concepts were codes openly, and then categorized and compared and related together by axial coding. Finally, the categories and their relations were purified based on the intended theory framework, through selective coding. The analysis process was assisted by using MAXQDA 10 software.

\subsection{Reflexivity}

The leading author (JB) is a medical doctor with ten years of experience in disaster and emergency management. He has worked as an EMS manager in one of the medical universities and is currently a $\mathrm{PhD}$ student in Health in Disasters and Emergencies. Hence, he is familiar with the current management system of mass casualty incidents in Iran.

\subsection{Ethical consideration}

The participants were ensured about confidentiality of their identity. They were briefed verbally and in writing, about the study, recording interviews, and the method of conducting the interview. In addition, they signed an informed consent letter. The study is a part of a dissertation with an ethical permission from Ethics Committee of Iran University of Medical Sciences (No: 94/D/105/4886; dated: 28 Oct. 2014).

\section{Results}

Two themes and seven sub-themes were developed as follows (Table 2):

Table 2. Themes and simple codes extracted from interviews

\begin{tabular}{|c|c|c|}
\hline Theme & Sub-theme & Code \\
\hline \multirow[t]{9}{*}{$\begin{array}{l}\text { The barrier } \\
\text { factors }\end{array}$} & \multirow[t]{3}{*}{ Multiplicity of relief agencies } & $\begin{array}{l}\text { Several organizations are involved in managing these } \\
\text { events. }\end{array}$ \\
\hline & & The accident scene is managed by several organizations. \\
\hline & & $\begin{array}{l}\text { In most accidents involve at least three or four of } \\
\text { emergency agencies. }\end{array}$ \\
\hline & \multirow[t]{2}{*}{ Lack of clear roles } & Limit set of organizational tasks is not defined. \\
\hline & & There is no clear description of personnel's job. \\
\hline & \multirow{2}{*}{$\begin{array}{l}\text { Lake of centralized and } \\
\text { integrated commanding }\end{array}$} & There is no a certain Commander. \\
\hline & & Currently we do not have a unified command. \\
\hline & \multirow[t]{2}{*}{ Cultural factors } & Citizens do not adhere traffic rules. \\
\hline & & We have a traditional management culture in Iran. \\
\hline \multirow[t]{6}{*}{$\begin{array}{l}\text { The facilitating } \\
\text { factors }\end{array}$} & \multirow[t]{2}{*}{$\begin{array}{l}\text { Number and distribution of EMS } \\
\text { posts }\end{array}$} & $\begin{array}{l}\text { Increasing the number of EMS posts has increased the } \\
\text { speed of our response. }\end{array}$ \\
\hline & & Emergency response time has decreased in recent years. \\
\hline & \multirow[t]{2}{*}{ Equipment and facilities } & Now we have good communication equipment. \\
\hline & & Our ambulances and personnel have tripled. \\
\hline & \multirow[t]{2}{*}{ Human force of the organizations } & $\begin{array}{l}\text { We have a good potential in the field of human resources, } \\
\text { especially in the pre-hospital. }\end{array}$ \\
\hline & & $\begin{array}{l}\text { The number of staff has increased in many organizations } \\
\text { over the past few years. }\end{array}$ \\
\hline
\end{tabular}

\subsection{Multiplicity of relief agencies}

The interviewees believed that the number of involved organizations is too much. The fire department, the Red Crescent, road services and even the private sector (cranes and trucks to remove vehicles from the scene), along with Emergency Medical Service (EMS) and the police are involved in managing special events that are demanding and somewhat beyond control. However, if there were just one or two organizations in charge of investigations, there would be more efficiency at the rescue operations. "... Most accidents involve three or four agencies such as: EMS, police, firefighters and the Red Crescent and this makes it difficult to establish interagency coordination..." (P 14). 


\section{2. Lack of clear roles among the involved first responder teams}

All participants agreed that lack of good internal and external coordination and specified roles among the organizations were the most important factor that affected the management of mass casualty traffic incidents adversely. Parallel and duplicate operations, failure to follow standard procedures, and several rescue and emergency phone numbers hindered cooperation and good teamwork. As a solution to improve coordination among the organization, some participants highlighted the role of the Emergency Operation Center (EOC) that can collect and share information from and among the aid organizations, connect these organizations together and call for back up considerations if needed.... The police keep ordering us about moving our vehicles, but not controlling the crowd. The fire department intervenes in our rescue tasks, by telling us that they should intervene first, to free the car passengers. The Red Crescent officers also believe they should be the first responders..." (P 1).

\subsection{Centralized and integrated command center at the scene}

Different people from different organizations with different task definitions attend mass casualty traffic incidents. If we cannot decrease the attending organizations, it is necessary to have one commander with the required practical and theoretical capabilities, equipment. According to the participants, there is no such person/party with adequate knowledge and insight about equipment and facilities of aid organizations and required authorities. They believed that "on-scene management" was the missing piece in the operation so that different organizations acted independently. "... Once, I asked a soldier to stop people from entering the scene and he said that he only took orders form the captain. Without any exaggeration, the incident scene is a real mess and there is no one to manage..." (P 2).

\subsection{Cultural factors}

Driving culture and laypeople's interest in intervening in the aid operations or gathering around the scene are some of the causes of mismanagement in operations. Such behavior at the incident scene is uncontrolled, not-managed, and mostly out of sheer emotions, which may create more problems in managing the scene of incident. Furthermore, lay people's attempts may include pulling out the injured people without considering safety measures that can result in irrevocable damages to the victim. Another aspect of culture was the public's hazardous driving and traffic behavior that may slow down the operation process through creating traffic jams around the incident venue and crash scene. "...There was a girl crying in the crash scene with torn clothes, she did not have her scarf on and many were filming her. The girl was bitterly crying and begging us to stop the people filming her. Some people tried to help her, lay people usually block our way to the incident place... (P 13).

\subsection{Number and distribution of EMS posts}

Timely arrival of EMS paramedics is highly effective in rescuing the injured or preventing permanent disabilities. The arrival time can be affected by the distance between EMS posts and the incident scenes. Then, proper geographical distribution of EMS posts is an influential way to minimize this time. According to the participants, rapid growth in the number of EMS posts has improved management of these incidents in recent years. There are 2,190 EMS posts nationwide in Iran, which can provide emergency medical services much faster. This development has resulted in decrease of death toll in mass casualty traffic incidents. "... One of our pivotal strengths is the increase in the number of EMS posts nationwide so that there are now many more operational EMS posts in the country than before. This can save lives of many injured in traffic incidents..." (P 5).

\subsection{Equipment and facilities to transport and provide health services to the injured}

Knowing that two thirds of deaths happen between the crash scene and hospital, availability of equipment such as cardiopulmonary resuscitation (CPR), equipment for faster transportation (standard ambulance and helicopter), and extrication tools can save many lives at the scene or on the way to the hospital. The majority of the participants admitted a great growth in available equipment and resources, so that improvement is visible in all aid organizations in terms of physical and operational facilities especially medical and transportation equipment. In addition, the personnel have managed to achieve higher levels of knowledge and operational skills standards. “...Ten years ago, we only had a few ambulances, which were actually old vans and Nissan Patrols or similar vehicles that were not suitable for transporting the injured. However, now we have much better, new ambulances with plenty of equipment that can help the injured on the way to hospital..." (P 5).

\subsection{Human force of the organizations}

Theoretically and practically capable personnel are highly effective in management of traffic incidents. The majority of the participants supported the idea of an increase in the number of aid and relief personnel and their knowledge over the last few years. This progress can result in an improvement in the quality of rescue services. In the past, 
there was no adequate well-trained workforce to provide advanced health care to save lives. But currently, nurses are present in most EMS posts who are able to provide complicated lifesaving services. "...A few years ago, just a driver, health assistant, and anyone available could be in charge of an EMS post. However, now we have technicians and physicians. We even have, helicopter and air rescue services pilots in our team. A sufficient amount of training workshops and conferences are held every year to improve our knowledge and skills. Nevertheless, there is a long way ahead to achieve the standards of advanced countries..." (P 4).

\section{Discussion}

According to the results, despite recent improvement in equipment, human resources and EMS posts, the process of investigation to mass casualty traffic incidents suffers from serious shortcomings. Khorasani et al. also stated the poor management of traffic incidents in Iran; although, they took all types of traffic incidents into account (23). Since more than a half of the deaths in traffic incidents happen at the crash scene or on the way to the hospital, and given that more than one-third ( 39 percent) of trauma deaths occur before arriving at the hospital, there is much to improve in the rescue process $(20,24,25)$. Improvement of onsite management in traffic crashes and rescue attempts on the way to the hospitals can be notably effective in decreasing death toll and disabilities. Finding an effective solution raised in our study entails cooperation and coordination among all the participating organizations and utilizing all equipment and capacities. The most effective factor, according to the result, was coordination among the organizations, which is not yet achieved because of several rescue call numbers, poor communication among the organizations, large extent of parallel and duplicate works, failure of sharing information, absence of "onsite management", ambiguity in tasks, and lack of standard procedures. Lack of coordination in management of traffic incidents and natural disasters and lack of onsite management have been mentioned by other authors as key managerial obstacles $(23,26)$. Establishment of an EOC can be a good way to create coordination among the organizations. Holgerson also concluded that absence of a centralized command system was one of the problems of onsite management in mass casualty traffic incidents (27). Lack of centralized command system at crash scene was mentioned by our participants as a managerial problem. By Iranian regulations, police are the authority in crash scenes; however, most of the participants argued that the police are not practically and theoretically capable of fulfilling this task because of lack of human resources, lack of adequate technical and specialized education and knowledge, and lack of adequate operational experience. Absence of a centralized command system in a crash scenes, in which different organizations participate, challenges coordination among the organizations and the opportunity to use all the capacities for an efficient management of incidents. Several organizations in Iran are in charge of dealing with incidents and this is a reason for poor coordination in rescue operations. Negative competition and contrast of interests among these organizations were also evident in our findings. Hence programming and coordinating police, rescue and aid teams, and EMS is an essential measure for managing mass casualty incidents, as suggested by others (27). The participants believed that having only one emergency and rescue phone number can coordinate the arrival time of different organizations and improve management of incidents. The participants highlighted physical and human resources as important factors effective in the improvement of services provided to the injured of mass casualty traffic incidents. Haghparast et al. also mentioned distribution and access to human resources, theoretical and practical incompetence of personnel, and lack of adequate resources as the causes of insufficient EMS (1). Kobsing et al. put emphasis on lack of equipment for transporting the injured and human resources as one of the challenges in the way of provided EMS in LMICs (28). Mack et al. also stated human and physical resources (equipment, tools, and infrastructures), organization, and management as three prevalent weaknesses in providing EMS and hospital healthcare services in developing nations (29). The participants believed that increasing pace of EMS services through increasing number and geographical expansion of EMS posts equipped with standard ambulances along with development of air rescue services had decreased the death toll and severity of injuries. On the other hand, by educating and training capable human forces and improving practical and theoretical capabilities of the personnel, more complicated and advanced services can be provided to the injured, on site and on the way to hospital and so decrease the death toll and severity of injuries. Laypeople's intervention in aid and rescue operation along with the troubles caused by crowd-gathering at the scenes can result in an increase of death toll. The intervention by laypeople might actually lead to death or permanent disability while they only want to help the injured. Khorasani et al. concluded that intervention by laypeople at crash scenes prolonged the aid personnel's arrival and secondary damages (23). Public education to not intervene in aid and rescue operations and simply giving first aid, such as controlling bleeding, if educated, and not to displace the injured can have positive effect (30). Moreover, improvement of the driving and traffic culture of the society through promoting proper driving behavior among public and private vehicle drivers is a way to prevent traffic incidents (31). Regarding the study limitations, there was no limitation except that in some cases, the interviews were interrupted by interviewees' customers, which might have distressed the interviewees and the interviewer. 


\section{Conclusions}

Optimum management of mass casualty traffic incidents can improve quality of services to the injured, and decrease the death toll. Such management requires the introduction of one emergency number and implementing and activating the EOC to improve convergence and coordination among organizations and to ensure utilization of all potentials. Increasing the number of EMS with optimum distribution and development of air EMS for faster and timely services are also recommended. Taking into account the key role of personnel of aid organizations, improving their theoretical and practical knowledge and skills can also lead to improvements. To this end, holding educational courses and introducing university programs and operational courses are recommended. Launching educational campaigns to improve public knowledge about first aid and driving culture are also recommended. Through such campaigns, people will be informed about the problems caused by unnecessary attendance of the public at the crash scene. Finally, providing adequate equipment is needed for providing advanced lifesaving services.

\section{Acknowledgments:}

This paper was part of a $\mathrm{PhD}$ thesis entitled "National model for effective management of traffic mass casualty incidents in I.R. Iran", supported financially by Iran University of Medical Sciences, through grant No. IUMS/SHMIS-1393/26.

\section{Conflict of Interest:}

There is no conflict of interest to be declared.

\section{Authors' contributions:}

All authors contributed to this project and article equally. All authors read and approved the final manuscript.

\section{References:}

1) Haghparast Bidgoli $\mathrm{H}$, Hasselberg $\mathrm{M}$, Khankeh $\mathrm{H}$, Khorasani-Zavareh D, Johansson E. Barriers and facilitators to provide effective pre-hospital trauma care for road traffic injury victims in Iran: a grounded theory approach. BMC emergency medicine. 2010; 10(1): 1. doi: 10.1186/1471-227X-10-20.

2) Björnstig U. Pre-Hospital Emergency Care In SWEDEN: with Special Emphasis on Care of Traffic Victims. IATSS research. 2004; 28(2): 24-31. doi: 10.1016/S0386-1112(14)60105-9.

3) Bakhtiyari M, Soori H. Epidemiology of traffic crashes outcomes and related factors. Journal of safety promotion and prevent injuries. 2013; 1(3): 150-9.

4) WHO. Global status report on road safety 2013: supporting a decade of action. World Health Organization; 2013.

5) Rezaei S, Arab M, Karami Matin B, Akbari Sari A. Extent, consequences and economic burden of road traffic crashes in Iran. J Inj Violence Res. 2014; 6(2): 57-63. doi: 10.5249/jivr.v6i2.191. PMID: 24045158, PMCID: PMC4009170.

6) Aitken P, Leggat P. Considerations in mass casualty and disaster management. InTech. 2012.

7) Herman J, Ameratunga S, Jackson R. Burden of road traffic injuries and related risk factors in low and middle-income Pacific Island countries and territories: a systematic review of the scientific literature (TRIP 5). BMC public health. 2012; 12(1): 1. doi: 10.1186/1471-2458-12-479.

8) Razzak JA, Shamim MS, Mehmood A, Hussain SA, Ali MS, Jooma R. A successful model of Road Traffic Injury surveillance in a developing country: process and lessons learnt. BMC public health. 2012; 12(1): 357. doi: 10.1186/1471-2458-12-357.

9) Gorji A. The first international congress of road safety in Mashhad, Iran. J Inj Violence Res. 2012; 4(2): 101-2. doi: 10.5249/jivr.v4i2.264. PMID: 22991699, PMCID: PMC3426899.

10) Lankarani KB, Mahmoodi M, Heydari ST, Joulaei H, Ghaffarpasand F, Maharlouei N, et al. The second international conference on reducing burden of traffic accidents, Shiraz, Iran. J Inj Violence Res. 2013; 5(1): 75-6. doi: 10.5249/jivr.v5i1.322. PMID: 23275241, PMCID: PMC3591734.

11) Organization WH. Mass casualty management systems: strategies and guidelines for building health sector capacity. 2007.

12) Leow J, Brundage S, Kushner A, Kamara $T$, Hanciles E, Muana A, et al. Mass casualty incident training in a resource - limited environment. Br J Surg. 2012; 99(3): 356-61. doi: 10.1002/bjs.7762. PMID: 22190046.

13) Saadat S, Yousefifard M, Asady H, Moghadas Jafari A, Fayaz M, Hosseini M. The most important causes of death in Iranian population; a Retrospective Cohort Study. Emerg (Tehran). 2015; 3(1): 16-21. PMID: 26512364, PMCID: PMC4614603. 
14) khankeh HR, co-authors. Disaster Hospital Prepardness. 2, editor. Iran: University of Welfare and Rehabilitation Sciences; 2013.

15) Education MoHaM. Reportable special events. In: Centre DaEM, editor. Iran: Disaster and Emergency Management Centre; 2015.

16) Emergency and disaster management center. Occurance 11.5 MCI in IRAN daily. 2015. Available from: http://isna.ir/fa/news/93103016907.

17) Bigdeli M, Khorasani Zavareh D, Mohammadi R. Pre-hospital care time intervals among victims of road traffic injuries in Iran. A cross-sectional study. BMC public health. 2010; 10(1): 1. doi: 10.1186/14712458-10-406.

18) Peden M, Scurfield R, Sleet D, Mohan D, Hyder AA, Jarawan E, et al. World report on road traffic injury prevention. Geneva: World Health Organization; 2004.

19) Tscherne H. The classic. Review article: Traffic accidents. 1966. Clin Orthop Relat Res. 2013; 471(9): 2755-9. doi: 10.1007/s11999-013-3012-9. PMID: 23633185, PMCID: PMC3734421.

20) Coats T, Davies G. Prehospital care for road traffic casualties. BMJ. 2002; 324(7346): 1135-8. doi: 10.1136/bmj.324.7346.1135. PMID: 12003887, PMCID: PMC1123093.

21) Strauss A, Corbin J. Basics of qualitative research: Techniques and procedures for developing grounded theory: Sage Publications; 1998.

22) Al-Busaidi ZQ. Qualitative research and its uses in health care. Sultan Qaboos Univ Med J. 2008; 8(1): 11 9. PMID: 21654952, PMCID: PMC3087733.

23) Khorasani-Zavareh D, Khankeh HR, Mohammadi R, Laflamme L, Bikmoradi A, Haglund BJ. Post-crash management of road traffic injury victims in Iran. Stakeholders' views on current barriers and potential facilitators. BMC Emerg Med. 2009; 9(1): 8. doi: 10.1186/1471-227X-9-8. PMID: 19435521, PMCID: PMC2692975.

24) Lockey D. Prehospital trauma management. Resuscitation. 2001; 48(1): 5-15. doi: 10.1016/S03009572(00)00313-0.

25) Hussain L, Redmond A. Are pre-hospital deaths from accidental injury preventable? BMJ. 1994; 308(6936): 1077-80. doi: 10.1136/bmj.308.6936.1077. PMID: 8173428, PMCID: PMC2539951.

26) Khankeh HR, Mohammadi R, Ahmadi F. Health Care Services at Time of Natural Disasters: A Qualitative Study. Iran Journal of Nursing. 2008; 51: 85-96.

27) Holgersson A. Review of on-scene management of mass-casualty attacks. Journal of Human Security. 2016; 12(1): 91-111. doi: 10.12924/johs2016.12010091.

28) Kobusingye OC, Hyder AA, Bishai D, Hicks ER, Mock C, Joshipura M. Emergency medical systems in low-and middle-income countries: recommendations for action. Bull World Health Organ. 2005; 83(8): 626-31. doi: 10.1590/S0042-96862005000800017. PMID: 16184282, PMCID: PMC2626309.

29) Mock C, Arreola-Risa C, Quansah R. Strengthening care for injured persons in less developed countries: a case study of Ghana and Mexico. Inj Control Saf Promot. 2003; 10(1-2): 45-51. doi: 10.1076/icsp.10.1.45.14114. PMID: 12772485.

30) Tiska M, Adu-Ampofo M, Boakye G, Tuuli L, Mock C. A model of prehospital trauma training for lay persons devised in Africa. Emerg Med J. 2004; 21(2): 237-9. doi: 10.1136/emj.2002.002097. PMID: 14988361, PMCID: PMC1726295.

31) Motevalian A. Road safety and traffic medicine in Iran: Achievements and challenges. J Local Glob Health Sci. 2015; 2015(2): 88. doi: 10.5339/jlghs.2015.itma.88. 